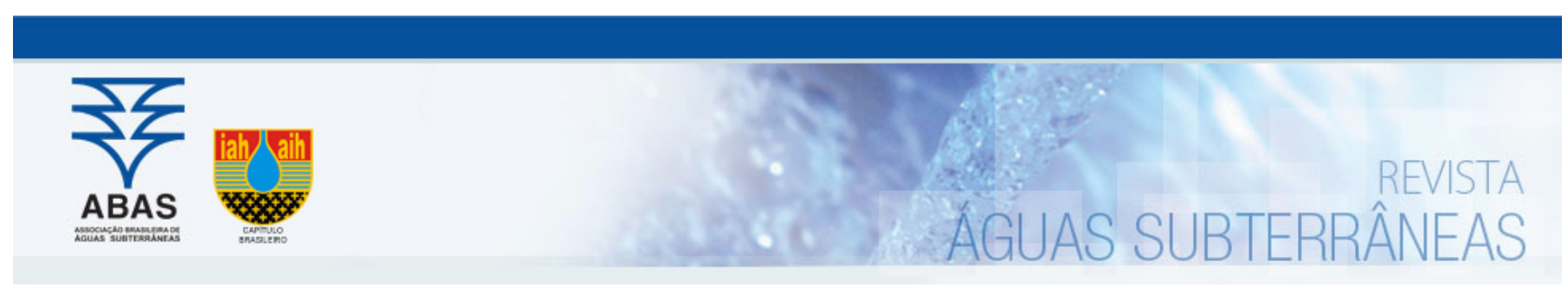

Artigos

\title{
Derivação de parâmetros para o uso do modelo SWAT na estimativa de recarga subterrânea em um aquifero cárstico- fissural do semiárido brasileiro
}

\section{Derivation of parameters for the use of the SWAT in the groundwater recharge estimate in a karst-fissural aquifero in brazil semiarid}

\author{
Lorrayne Silva1; Stela Dalva dos Santos Cota ${ }^{\square}$ \\ ${ }^{1}$ Centro de Desenvolvimento da Tecnologia Nuclear - CDTN/CNEN, Belo Horizonte, Minas Gerais
}

$\triangle \underline{\text { lorrayne.geo@gmail.com, stelacota@gmail.com }}$

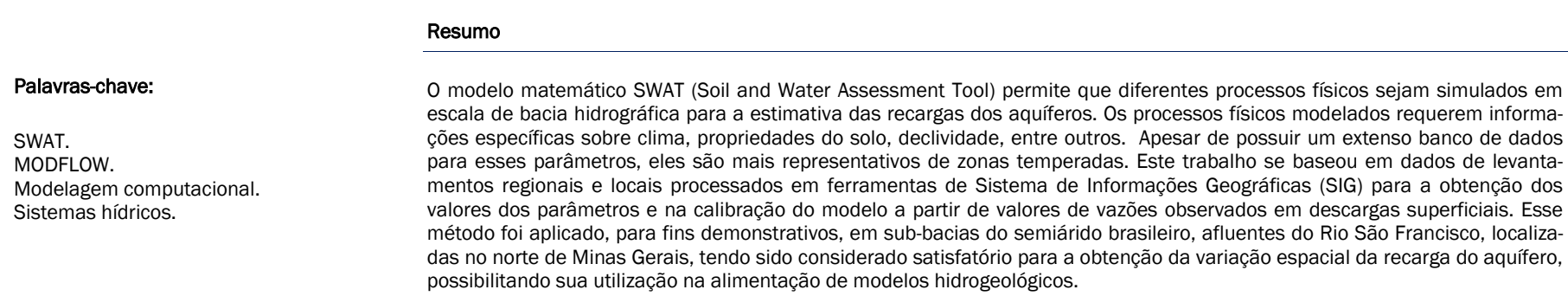

Keywords:

Abstract

SWAT.

MODFLOW.

Computacional modeling.

Hydrologic systems.

Revisado por pares.

Aprovado em: 10/12/2018.

\begin{abstract}
The mathematical model SWAT (Soil and Water Assessment Tool) allows different physical processes to be simulated in a watershed scale to estimate the aquifer recharge rates. The modeled physical processes require specific information about climate, soil properties, slope, among others. The model has a comprehensive database for these parameters, but they are more appropriated to temperate zones. This work applied regional and local data using Geographic Information System (GIS) tools to obtain the parameter values and the calibration of the model using observed surface discharge rates. This method was applied for demonstration purposes in watersheds of the Brazilian semi-arid, tributaries of the São Francisco River, located in the north of Minas Gerais, and was considered satisfactory for capturing the spatial variability of the recharge rates of the aquifer, allowing their use in hydrogeological models.
\end{abstract}

DOI: http:/dx.doi.org/10.14295/ras.v33i1.29160

\section{INTRODUÇÃO}

O modelo matemático SWAT (Soil and Water Assessment Tool) permite que diferentes processos físicos sejam simulados em escala de bacia hidrográfica. Esse modelo é capaz de analisar os impactos das alterações no uso do solo sobre o escoamento superficial e subterrâneo, sobre a produção de sedimentos e sobre a qualidade da água em bacias hidrográficas rurais e urbanas (NEITSCH et al., 2011). De particular interesse para o estudo dos processos subterrâneos está sua capacidade de estimar as recargas dos aquíferos. Este parâmetro é parte integrante do modelo hidrogeológico conceitual de um sistema aquífero e é primordial para a elaboração de modelos de fluxo subterrâneo.
Diversos trabalhos têm sido desenvolvidos utilizando o modelo SWAT agregado a modelos de fluxo subterrâneo, principalmente o modelo MODFLOW (McDonald e Harbaugh, 1983), para a caracterização de sistemas hidrogeológicos. Nesses trabalhos, o SWAT é utilizado, de forma sequencial ou integrada ao MODFLOW, para a estimativa das recargas. Como um exemplo, Wible (2014) propôs a criação de uma ligação espacial generalizada entre SWAT e MODFLOW, tendo sido aplicado na porção norte do rio Sprangue, no alto da bacia Klamath, em Oregon. Também cita-se Dowlatabadi e Zomorodian (2015), que realizaram uma simulação conjunta para as águas superficiais e subterrâneas utilizando os modelos SWAT e MODFLOW e aplicado na estimativa da taxa de recarga na bacia agrícola do Firoozabad, no Irã. 
O componente hidrológico do modelo SWAT inclui sub-rotinas de escoamento superficial, infiltrações nos solos, fluxo lateral superficial, escoamento de base, recarga para o aquífero livre (PERC), recarga do aquífero confinado e evapotranspiração (NEITSCH et al., 2011). Destaca-se que a percolação para o aquífero livre é o principal componente de interesse para o presente estudo. A Equação 1 apresenta a equação de equilíbrio hídrico, conforme simulado pelo SWAT.

$$
S W_{i}=S W+\sum_{i=1}^{t}\left(R_{i}-Q_{i}-E T_{i}-P_{i}-Q R_{i}\right)
$$

Onde: SWi é a quantidade final de água no solo (mm), SW é a umidade inicial do solo no dia $i(\mathrm{~mm}), t$ é o tempo (dias), $R_{i}$ é a quantidade de água que entra na zona de evapotranspiração no dia $i$ $(\mathrm{mm}), Q_{i}$ é a quantidade de escoamento superficial no dia $i(\mathrm{~mm})$, $E T_{i}$, é a quantidade de evapotranspiração no dia $i(\mathrm{~mm}), P_{i}$ é a quantidade de água que entra na zona de aeração do perfil do solo (percolação) no dia $i(\mathrm{~mm})$, e $Q R_{i}$ é a quantidade de retorno do fluxo no dia $i(\mathrm{~mm})$.
O modelo SWAT simula os processos superficiais e subsuperficiais baseados em Unidades Hidrológicas de Resposta (UHRs), que são unidades conceituais de uso, manejo, declive e características homogêneas do solo (ARNOLD et al., 1998). As UHRs são modeladas como representações não geolocalizadas, espacialmente desconectadas de polígonos e pertencentes a uma dada sub-bacia no domínio de superfície.

As equações dos processos físicos associados à movimentação hídrica requerem, como dados de entrada, informações específicas sobre clima, propriedades do solo, topografia, entre outras. Em relação às dificuldades encontradas para a aplicação do modelo a bacias hidrográficas brasileiras, destaca-se a escassez de valores apropriados no banco de dados do SWAT (BRESSIANI et al., 2015). Dentro deste contexto, o objetivo deste trabalho é desenvolver caminhos possíveis para a obtenção dos valores dos parâmetros necessários para a execução do modelo SWAT aplicado à realidade brasileira, em especial para bacias do semi-árido, tendo sido aplicado, para fins demonstrativos, em bacias hidrográficas afluentes do Rio São Francisco, localizadas no norte de Minas Gerais.

Figura 1 - Desenho esquemático dos processos hidrológicos que compõem o modelo SWAT

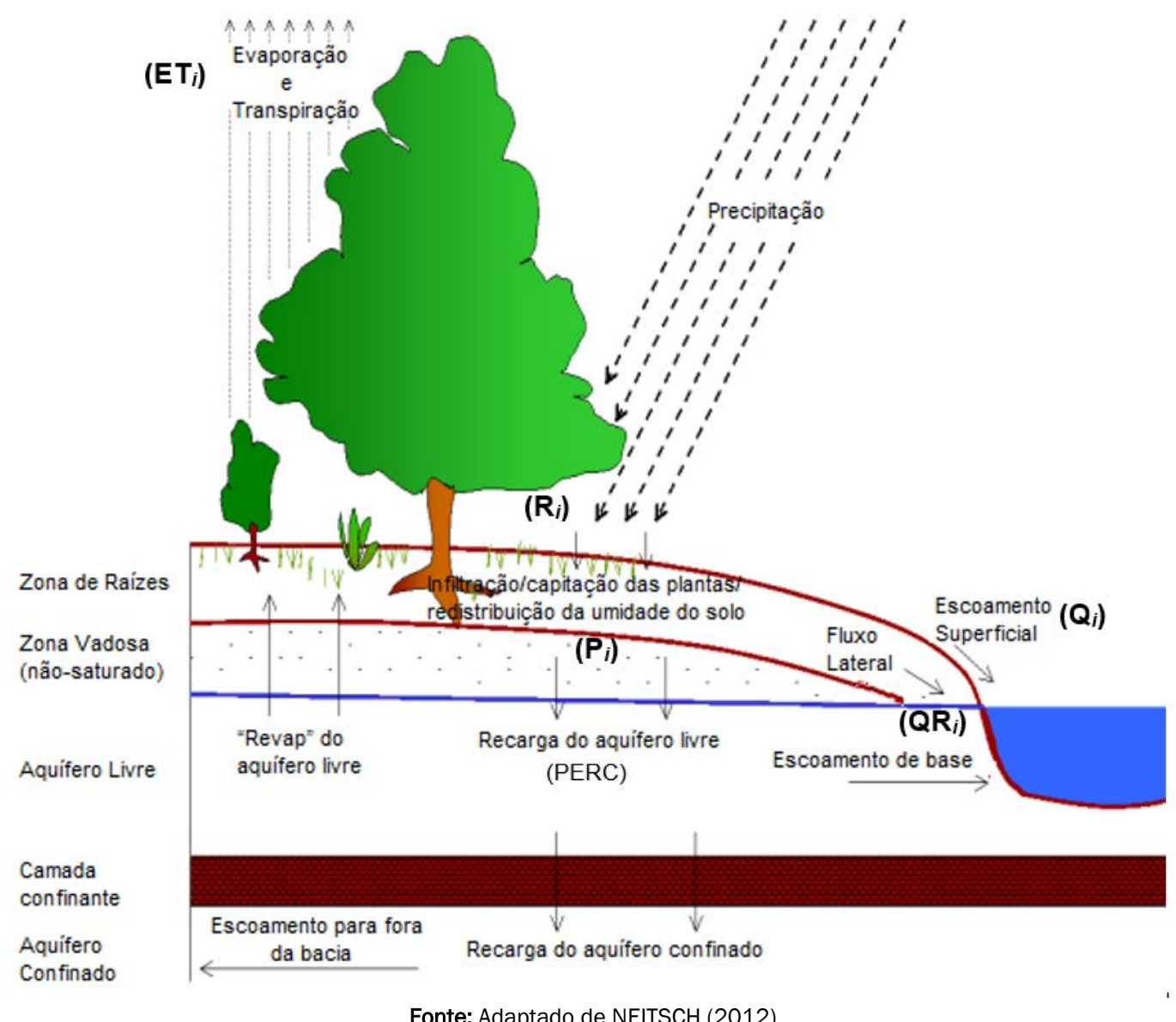

Fonte: Adaptado de NEITSCH (2012) 


\section{METODOLOGIA}

\subsection{Informações sobre a área}

A área de estudo encontra-se na margem oriental do Vale do Rio São Francisco, ocupa aproximadamente $574 \mathrm{~km}^{2}$. Envolve as bacias hidrográficas dos riachos Boi Morto, Tabocas e Mocambo, afluentes do médio curso do rio São Francisco situados no município homônimo, região norte do Estado de Minas Gerais (Figura 2).

Desde o início dos anos 2000, vários estudos foram desenvolvidos na área, conduzidos por pesquisadores do Instituto de Geociências da Universidade Federal de Minas Gerais (UFMG) e em parcerias com o Centro de Desenvolvimento da Tecnologia Nuclear (CDTN) e o Centro Federal de Educação Tecnológica de Minas Gerais (CEFET-MG), entre eles citam-se Freire e Castro (2003), Velásquez et al. (2003), Velásquez et al., (2006); Moura e Souza (2010), Cota (2011) e De Paula (2012).
A área insere-se no contexto geológico da Bacia Sedimentar do São Francisco, que corresponde à porção meridiana do cráton homônimo (ALKMIM e MARTINS-NETO, 2001). A região está caracterizada por uma sucessão de rochas neoproterozoicas, correspondentes ao Grupo Bambuí, sendo identificadas, da base para o topo, as formações Serra de Santa Helena, Lagoa do Jacaré e Serra da Saudade, além de rochas cretáceas do Grupo Urucuia e coberturas cenozoicas.

A área de estudo é constituída pelas sub-bacias dos riachos Mocambo, Tabocas, Pageú, Várzea de Casa, Tinguis, Boi Morto, de um riacho sem nome, a oeste da área, e pequenos córregos próximos à sede urbana de São Francisco (Figura 3). Durante os trabalhos realizados por Cota (2011), foram instalados dois transdutores de pressão nas seções fluviais de jusante dos riachos Mocambo e Tabocas, revelando o caráter intermitente destes cursos d'água (Tabela 1).

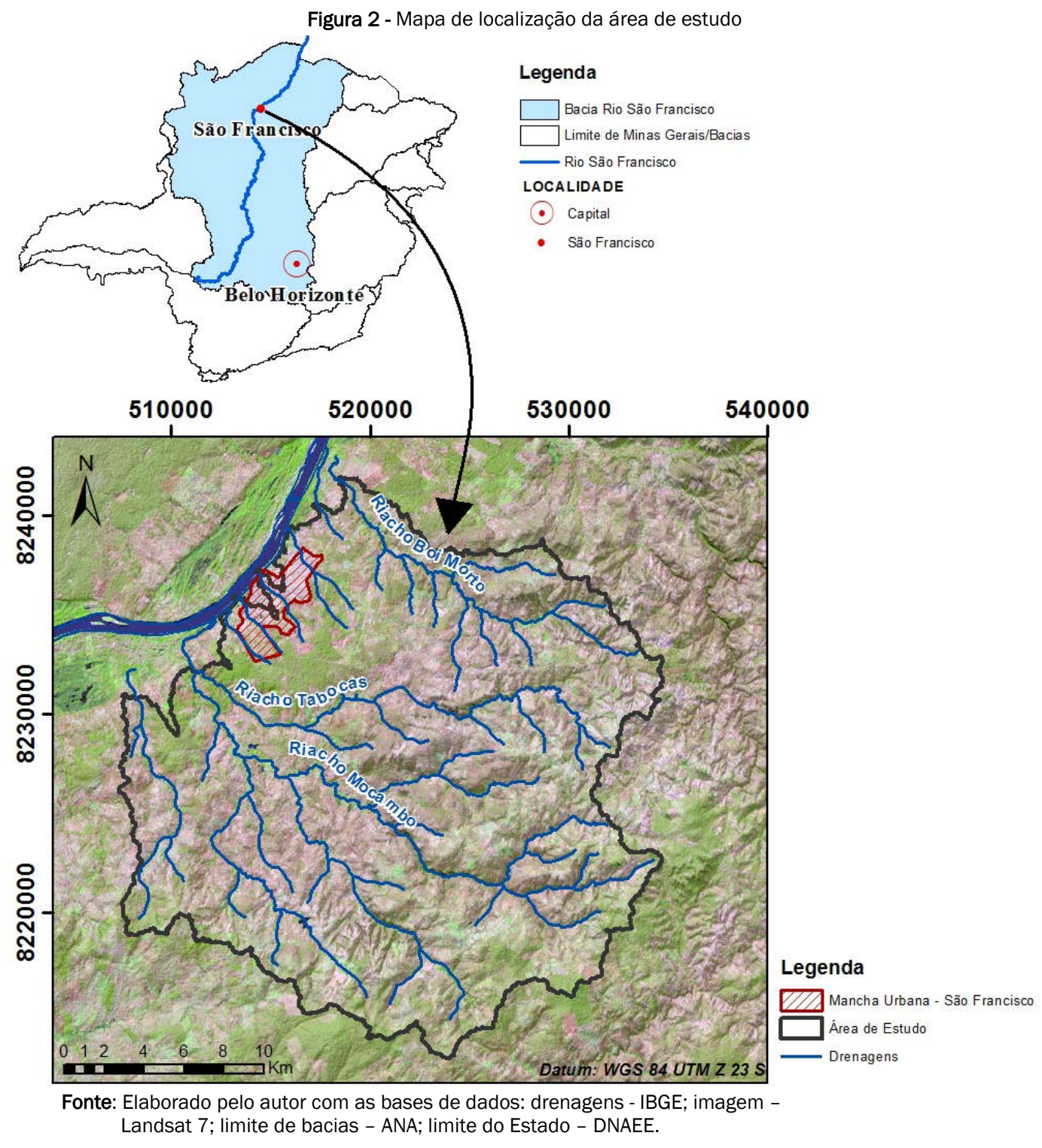


As vazões específicas médias de longo período das sub-bacias do rio São Francisco, dentro do limite da área de estudo, foram determinadas pelo estudo de regionalização de vazões para bacias de até $500 \mathrm{~km}^{2}$ (UFV, 2005), sendo as vazões específicas médias entre 2 e $5 \mathrm{~L} / \mathrm{s} . \mathrm{km}^{2}$.

Tabela 1 - Estações de monitoramento fluviométrico

\begin{tabular}{|c|c|c|c|c|}
\hline Ponto de Monitoramento & UTM E (m) & UTM N (m) & $\begin{array}{c}\text { Área de Drenagem } \\
\left(\mathrm{km}^{2}\right)\end{array}$ & Período de Monitoramento \\
\hline Flu 1 (Mocambo) & 511.070 & 8.231 .713 & 269,3 & Out/2009 a Mar/2010 \\
\hline Flu 2 (Tabocas) & 511.605 & 8.232 .161 & 73,2 & Out/2009 a Mar/2010 \\
\hline
\end{tabular}

Figura 3 - Localização dos instrumentos de monitoramento fluviométrico

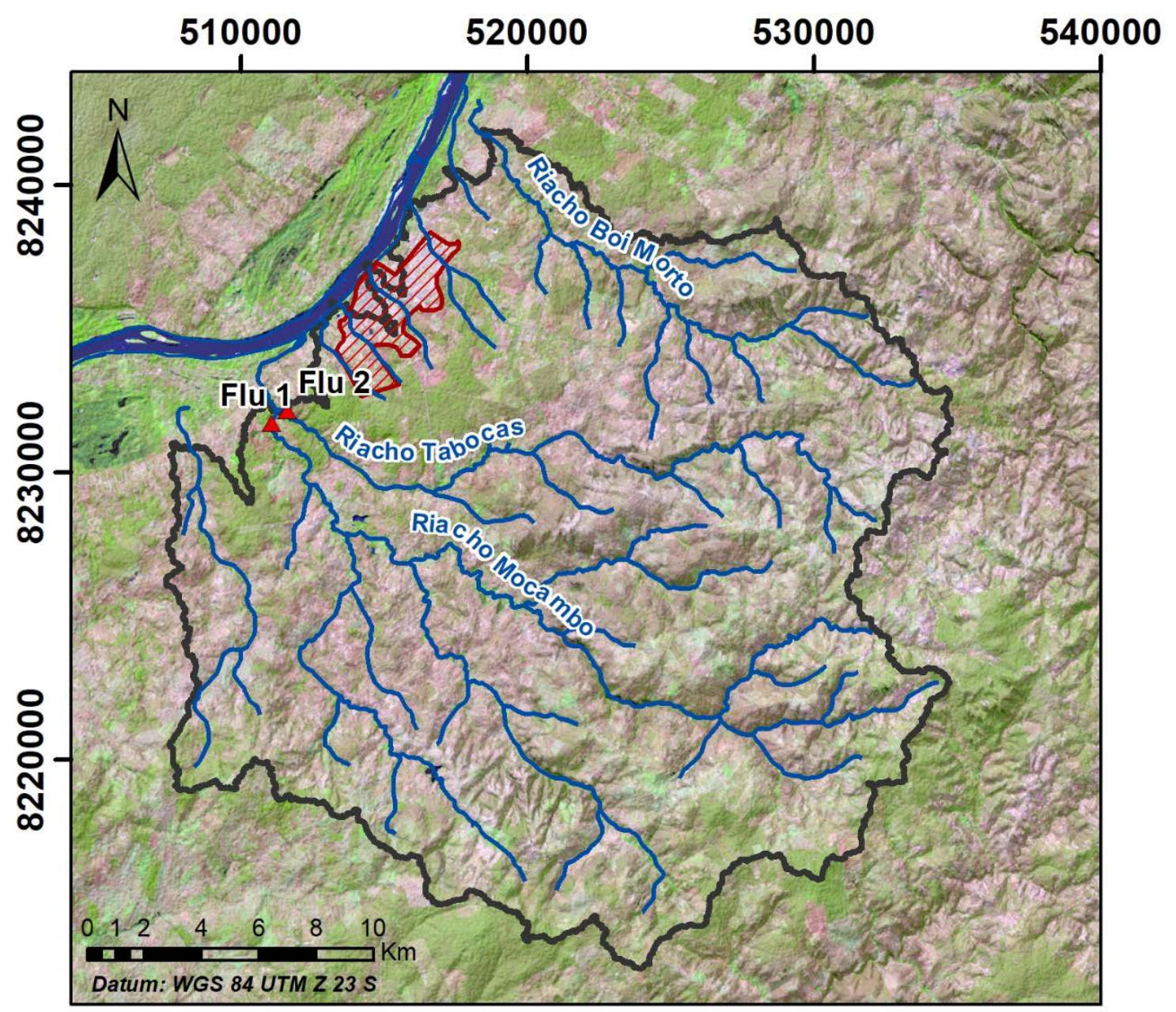

Fonte: Elaborado pelo autor com as bases de dados: drenagens - IBGE; imagem - Landsat 7.

Durante os trabalhos realizados por Cota (2011) e De Paula (2012), foram instalados pluviômetros nas cabeceiras dos riachos Mocambo e Boi Morto; no entanto, trata-se de séries restritas e com muitas falhas de leituras. Desta forma, optou-se por utilizar estações com dados disponíveis no entorno da área de estudo. Apenas duas estações no entorno da área apresentaram dados até o presente. A estação 1544012, no município de São Francisco (Plu-1), na porção mais baixa da área de estudo; e a estação 1644027, no município Brasília de Minas (Plu-2), na porção alta da área. Tabela 2 são apresentadas a precipitação média de cada pluviômetro para o período do ano hidrológico monitorado.

Tabela 2 - Localização e precipitação média nos pluviômetros

\begin{tabular}{ccccc}
\hline Ponto & UTM E $(\mathrm{m})$ & UTM N $(\mathrm{m})$ & Referência & $\begin{array}{c}\text { Precipitação } \\
\text { Mérie Monitorada (mm/ano) }\end{array}$ \\
\hline Plu-1 & 514.115 & 8.236 .657 & ANA - 1544012 & $1940-2015$ \\
Plu-2 & 561.483 & 8.209 .143 & ANA - 1544027 & $1975-2015$ \\
\hline Fonte: Elaborado pelo autor & &
\end{tabular}

Fonte: Elaborado pelo autor 
Portanto, a chuva média diária na área de estudo foi estabelecida pela média das chuvas diárias nas duas estações ANA. A partir dos resultados obtidos foi verificada a semelhança com os dados de chuvas diárias existentes nos pluviômetros instalados por Cota (2011) e De Paula (2012).A conceitualização da dinâmica hídrica proposta por Cota (2011) e De Paula (2012) tem como base comum os seguintes componentes (Figura 4): a) 0 fluxo nos aquífe- ros é essencialmente horizontal, com exceção do fluxo vertical nas áreas de recarga; b) o principal mecanismo de entrada de água é a recarga; c) a região de descarga é representada pela rede de drenagem dos riachos Boi Morto, Tabocas e Mocambo e, principalmente, pelo rio São Francisco; d) os limites do fluxo subterrâneo na bacia foram baseados em limites físicos topográficos e na análise da potenciometria.

Figura 4 - Representação esquemática do modelo hidrogeológico conceitual da área de estudo

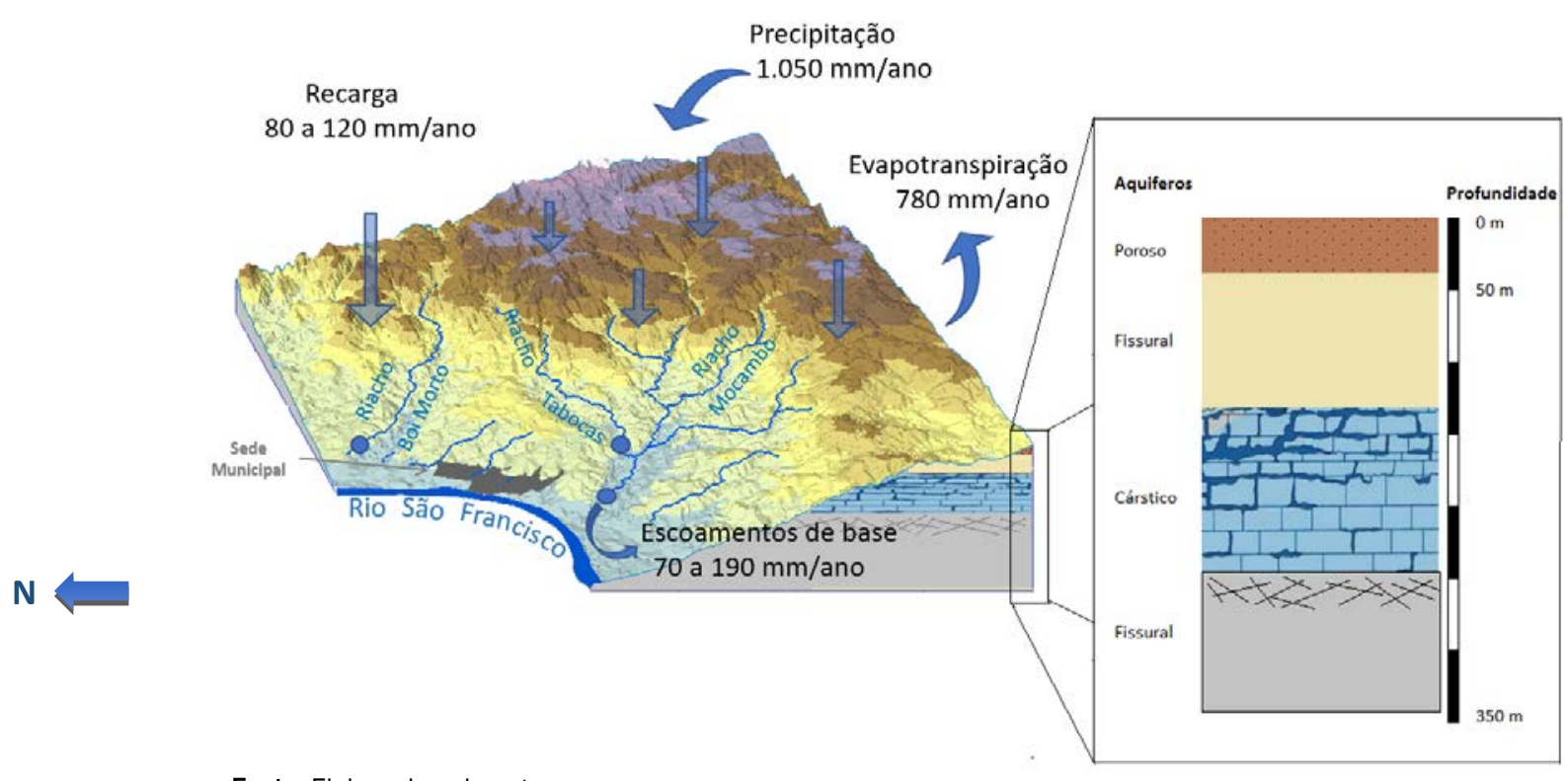

Fonte: Elaborado pelo autor

\subsection{Levantamento de informações específicas para o modelo SWAT}

Os processos físicos associados ao movimento de água, modelados pelo SWAT, requerem informações específicas sobre clima, propriedades do solo, topografia, declividade, vegetação e as práticas de manejo do solo. Essas informações foram compiladas a partir de levantamentos regionais e locais e processadas em uma base de Sistema de Informações Geográficas (SIG) utilizando o software ArcGIS $^{\circledR}$ e planilhas.

Na elaboração do mapa de uso e ocupação do solo foi utilizada a classificação supervisionada de uma imagem LANDSAT 7, com resolução espacial de 15 metros e datada de abril de 2010 (Figura 5a). Foram criadas cinco classes de uso do solo, a saber: área urbanizada, várzea, área de pastagem, área de vegetação nativa e área de solos expostos (Figura 5b). O Modelo Digital de Elevação (MDE) foi obtido por meio do endereço eletrônico http://earthexplorer.usgs.gov/, disponibilizado pelos Serviços Geológicos dos Estados Unidos, com resolução de $30 \times 30$ metros (Figura 5c). 0 mapa de solos foi originado a partir do levantamento de solos no Estado de Minas Gerais publicado pela FEAM (2010) na escala de 1:650.000. Na área de estudo foram encontradas 3 classes de solo: cambissolo, latossolo e neossolos (Figura 5d).

O modelo SWAT requer um grande número de parâmetros físicohídricos do solo. Em termos de origem geológica e geomorfológica, os solos brasileiros diferem daqueles formados em regiões temperadas, como a Europa e os EUA, os quais compõem o banco de dados do SWAT. Desta forma, foi necessária a criação do banco de dados locais para a realização das simulações. 
Figura 5 - a) Imagem LANDSAT 7; b) mapa de uso e ocupação do solo; c) Modelo Digital de Elevação (MDE); d) mapa de solos (FEAM, 2010)
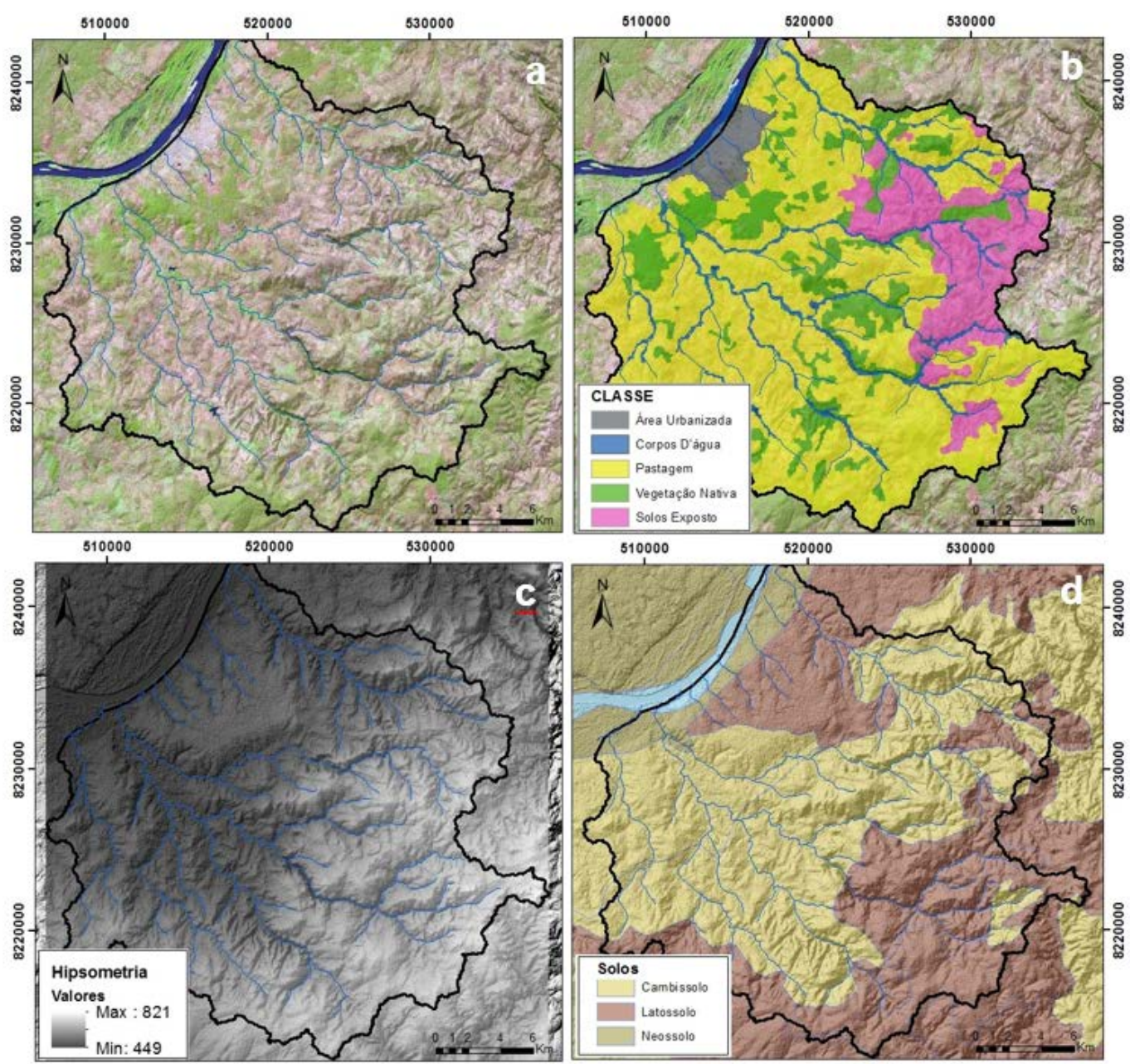

Fonte: Elaborado pelo autor

Para a determinação dos dados climáticos (umidade relativa do ar, temperatura, evaporação, irradiação etc.), foram levantadas as estações climatológicas operantes pelo Instituto Nacional de Meteorologia (INMET) mais próximas da área, tendo sido selecionadas as estações de São Francisco (código 83385), Januária (código 83386) e Montes Claros (código 83437).

Os dados climáticos, como precipitação diária, temperatura do ar, radiação solar, velocidade do vento e umidade do ar, são requeridos como dados de entrada pelo SWAT. Esses dados podem ser os monitorados no local ou gerados por um código do programa. Para a geração de dados climáticos são necessários dados estatísticos com representatividade climática das bacias, portanto, no manual (ARNOLD et. al., 2012) é recomendado estatísticas de monitoramento de pelo menos 20 anos.

Os dados de vazão de escoamento são muito importantes, uma vez que possibilitam a adequação dos parâmetros calibráveis à realidade da bacia na qual o modelo está sendo aplicado. Portanto, as vazões de descarga calculadas previamente por Cota (2011) e De Paula (2012) nas bacias dos riachos Mocambo, Taboca e Boi Morto foram consolidadas e reavaliadas neste estudo.

Desta forma, os valores de níveis de coluna de água, coletados em campo por transdutores de pressão, foram convertidos em vazões por meio do método de Manning (1891). Para aplicação da referi- da metodologia utilizou-se o levantamento topobatimétrico das seções e dos perfis longitudinais dos cursos d'água, obtidos por Cota (2011) e De Paula (2012).

Os valores do parâmetro de rugosidade da superfície dos rios foram estimados pelos autores consultando a bibliografia e, validados neste estudo, consultando a tabela de referências elaborada por Chow (1959). Os valores adotados para as bacias dos riachos Mocambo e Tabocas foi de 0,040 e 0,035, respectivamente; já na bacia do riacho Boi Morto adotou-se o valor de 0,033, conforme descrito por De Paula (2012).

\subsection{Modelo SWAT}

O primeiro nível de subdivisão do modelo SWAT é denominado de sub-bacias. Estas possuem uma posição geográfica e são espacialmente relacionadas entre si. Foram delineadas 11 sub-bacias, obtidas pelos limites hidrográficos definidos por meio da superfície topográfica e da rede de drenagem (Figura 6). As sub-bacias são divididas em Unidades Hidrológicas de Resposta (UHRs), obtidas segundo os atributos de uso e ocupação, solo e declividade. Este modelo resultou em 247 combinações destes atributos.

Como regra geral apresentada pelo manual, uma dada sub-bacia deve ter entre 1 a 10 UHRs. Portanto, foram utilizados filtros disponíveis no software capazes de agrupar múltiplas UHRs. Desta 
forma, foram realizadas algumas provas e adotados os limites mínimos de área para o agrupamento das UHRs: $10 \%$ de uso e ocupação; $10 \%$ de classe do tipo de solo; e 30\% de classe de declividade, resultando em um total de 76 UHRs. Considerado um número satisfatório, uma vez que se esperava entre 11 e 110 UHRs.

As bacias brasileiras são pobres em informações que remetem aos parâmetros das propriedades físico-hídricas dos solos. Para auxiliar na determinação desses parâmetros, foi utilizado o programa computacional para a calibração automática e de análises de sensibilidade e incertezas dos parâmetros de entrada, o SWAT-CUP (i.e., SWAT - Calibration and Uncertainty Program) (ABBASPOUR et al., 2007). O SWAT-CUP é uma interface desenvolvida para agregar algoritmos de otimização (SUFI-2, GLUE, ParaSol, MCMC e PSO), facilitando as análises de sensibilidade, calibração, validação e análises de incertezas do modelo SWAT (PINIEWSKI, 2014).

A análise global de sensibilidade dos parâmetros neste trabalho foi realizada de forma automática, através do método ParaSol (DUAN et al., 1993). A calibração contemplou o período de 2009 a 2010, buscando ajustar as vazões observadas às vazões calibradas das bacias dos riachos Mocambo e Tabocas neste período, livres das instabilidades das condições iniciais. O coeficiente Nash-Sutcliffe (COE) foi utilizado como critério estatístico para avaliar o ajuste do modelo.

Figura 6 - Mapa da distribuição das sub-bacias na área de estudo

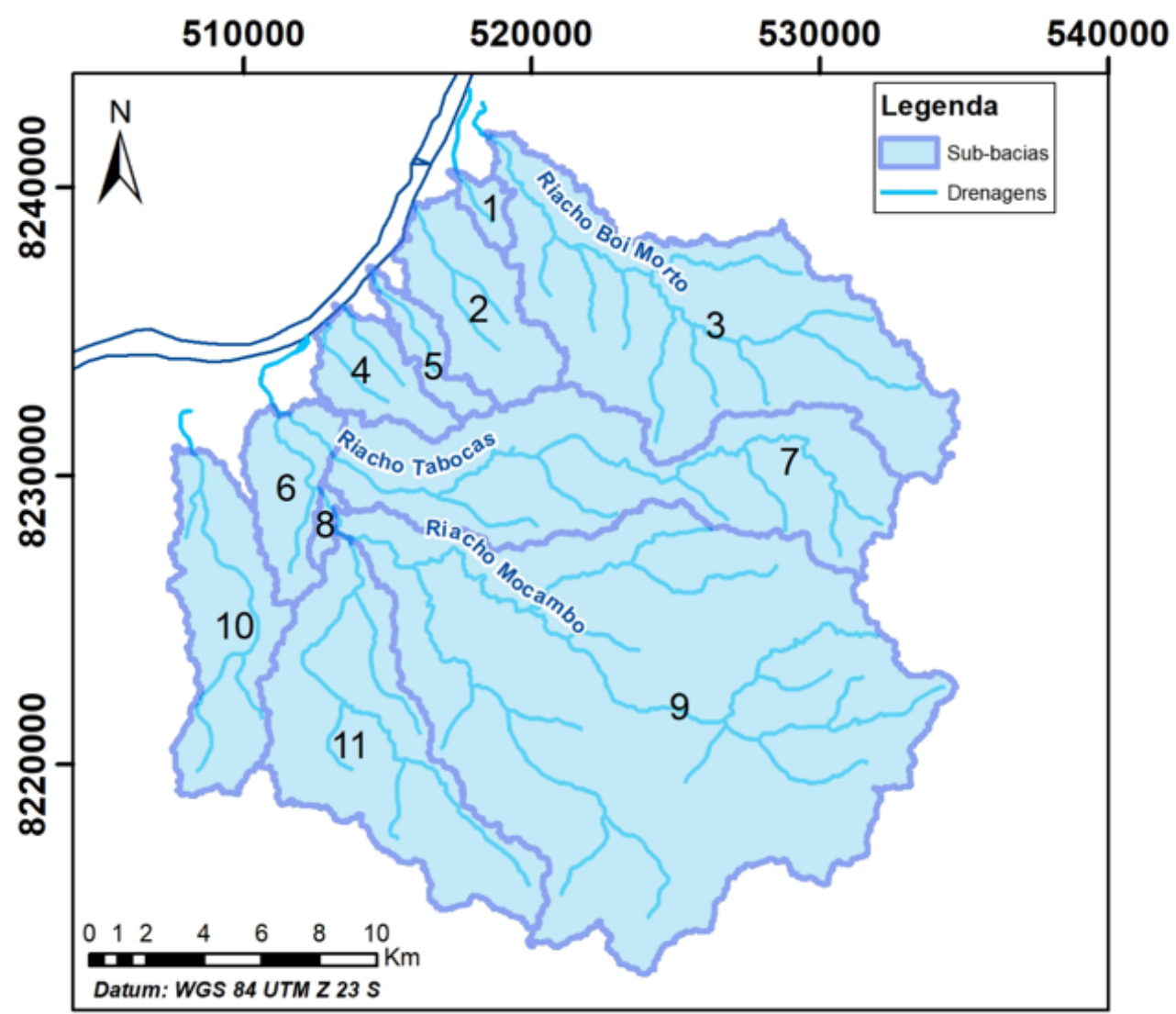

Nota: 1.Sub-bacia riacho sem nome, 2.Sub-bacia riacho sem nome, 3.Sub-bacia riacho Boi Morto, 4.Sub-bacia riacho sem nome, 5.Sub-bacia riacho sem nome, 6.Sub-bacia riacho Mocambo jusante do riacho Tabocas, 7.Sub-bacia riacho Tabocas, 8.Sub-bacia riacho Mocambo jusante do riacho Tinguis, 9.Sub-bacia riacho Mocambo, 10.Sub-bacia riacho sem nome, 11.Sub-bacia riacho Tinguis.

Fonte: Elaborado pelo autor

\section{RESULTADOS E DISCUSSÕES}

Para a obtenção das vazões, por meio do método de Manning (1891), foram traçadas as curvas chaves das bacias monitoradas, obtendo o polinômio de regressão.
A partir do polinômio da curva chave, os valores de níveis coletados em campo pelos transdutores foram convertidos em vazões. Como mencionado anteriormente, a chuva média diária na área de estudo foi estabelecida pela média das chuvas diárias em duas estações ANA. No gráfico da Figura 7, observa-se a distribuição das vazões com a precipitação. 


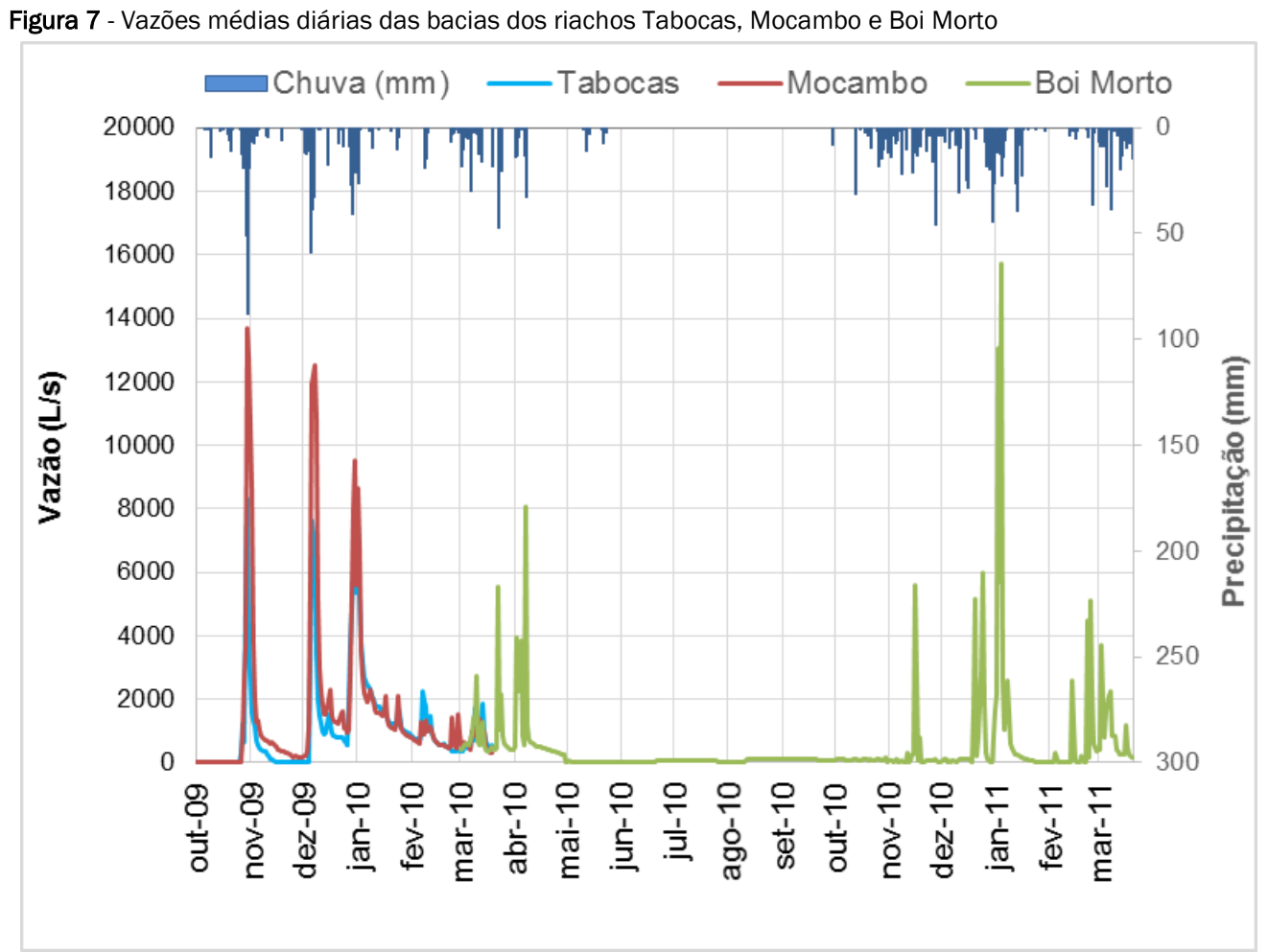

Fonte: Elaborado pelo autor

Como entrada do modelo SWAT, foram utilizadas estimativas para cada parâmetro associado às classes de solos, por meio de valores encontrados em referências a respeito dos solos brasileiros. Em geral, os valores foram levantados por Baldissera (2005). Os valores adotados, para a primeira camada de solo, estão na Tabela 3.

Os parâmetros calibráveis do modelo SWAT são classificados de duas formas: pelo "t-Stat", que fornece uma medida de sensibilidade em que os maiores valores absolutos são os mais sensíveis; e pelo "P-Value", no qual os valores próximos de zero são os que indicam maior significância da sensibilidade. Na Figura 8 é apresentada a classificação de sensibilidade dos parâmetros calibrados.

Observa-se na Figura 8 que o parâmetro que apresenta maior sensibilidade é o número de curva SCS (CN2), seguido pelos parâmetros coeficiente de armazenamento de escoamento de base (ALPHA_BNK), fator de compensação de evapotranspiração do solo (ESCO), coeficiente de evaporação das águas subterrâneas (GW_REVAP) e a constante de recessão do escoamento de base (ALPHA_BF).

Tabela 3 - Valores dos parâmetros físico-hídricos do solo

\begin{tabular}{|c|c|c|c|}
\hline Propriedades & Cambissolo & $\begin{array}{l}\text { Latossolo } \\
\text { Vermelho-Amarelo }\end{array}$ & Neossolos \\
\hline NLAYERS & 5 & 4 & 4 \\
\hline HYDROGRP & $\mathrm{B}$ & $\mathrm{B}$ & $\mathrm{B}$ \\
\hline $\begin{array}{l}\text { SOL ZMX (mm) } \\
\text { ANION_EXCL }\end{array}$ & $\begin{array}{c}1170 \text { (a) } \\
0,47\end{array}$ & $\begin{array}{c}1500 \\
0,38\end{array}$ & $\begin{array}{c}1400 \\
0,33\end{array}$ \\
\hline $\mathrm{SOL}$ CRK $\left(\mathrm{m}^{3} / \mathrm{m}^{3}\right)$ & 0,5 (a) & 0,498 (b) & $0,62^{(b)}$ \\
\hline $\begin{array}{l}\text { SOL_Z1 (mm) } \\
\left.\text { SOL_BD1 ( } / \mathrm{cm}^{3}\right)\end{array}$ & $\begin{array}{c}300 \\
1,6\end{array}$ & $\begin{array}{c}360 \\
1,2^{(b)}\end{array}$ & $\begin{array}{c}130 \\
0,947 \text { (b) }\end{array}$ \\
\hline SOL_AWC1 $(\mathrm{mm} / \mathrm{mm})$ & 0,15 & 0,3 & 0,14 (a) \\
\hline SOL_CBN1 (\% wt.) & 1,4 & 1,1 & 1,7 \\
\hline SOL_K1 (mm/h) & 12,5 & $40^{(b)}$ & $182^{(b)}$ \\
\hline CLAY1 (\% wt.) & 36,3 & 30,3 & 13,7 \\
\hline SILT1 (\% wt.) & 18 & 16 & 56,7 \\
\hline SAND1 (\% wt.) & 45,7 (a) & 53,7 & 29,6 \\
\hline ROCK1 (\% wt.) & 0 & 0 & 0 \\
\hline SOL_ALB1 (fraction) & 0,15 & 0,15 & 0,16 \\
\hline USLE_K1 & 0,05 & 0,13 & 0,16 \\
\hline SOL_EC1 (Ds/m) & 0 & 0 & 1 \\
\hline
\end{tabular}

Notas: (a) Ajustes; (b) Ribeiro et al., 2007.

Fonte: adaptado de Baldissera (2005) 
Figura 8 - Classificação crescente da sensibilidade dos parâmetros relacionados à vazão para a área de estudo

P-Value

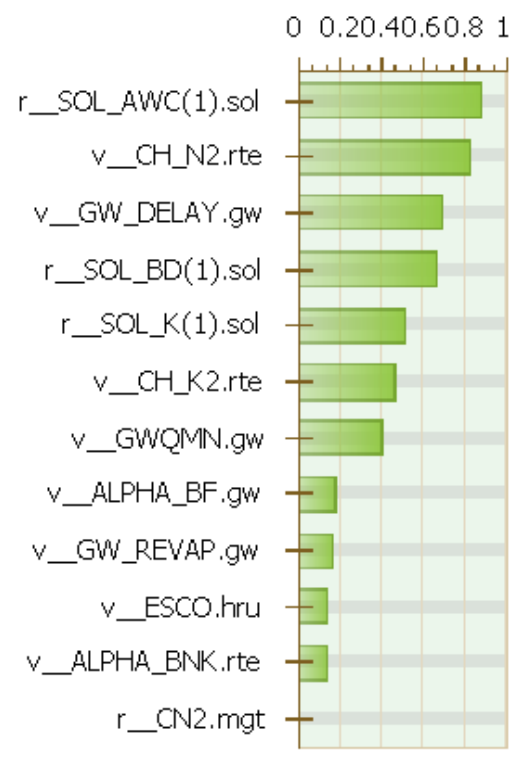

Fonte: Elaborado pelo autor

\section{t-Stat}

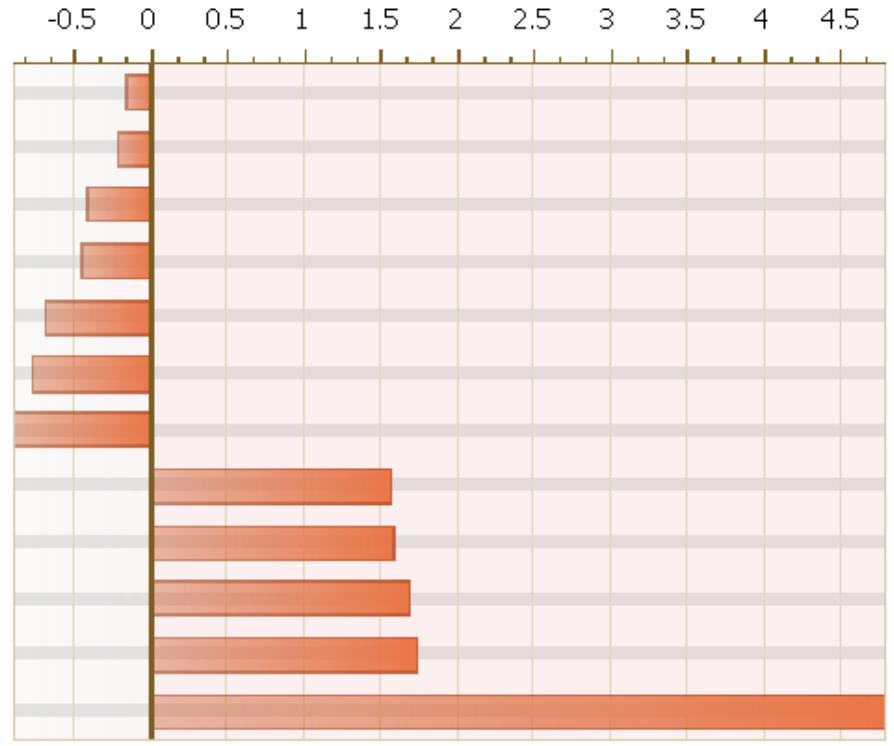

A faixa de variação utilizada para cada parâmetro e o respectivo valor calibrado encontram-se na Tabela 4. Com os valores dos parâmetros fixados como sendo os valores calibrados, executou-se o modelo para o período de outubro de 2009 a abril de 2010. As vazões diárias calibradas foram confrontadas com as vazões médias diárias observadas, conforme podem ser visualizadas na Figura 9 e na Figura 10, para a bacia do riacho Mocambo e Tabocas, durante o período chuvoso do ano hidrológico 2009-2010. A bacia do riacho Boi Morto não foi utilizada para a calibração, pois não há dados monitorados de vazão durante o período mencionado.
Na Figura 9 e na Figura 10 verificou-se que, de modo geral, o SWAT superestimou as vazões de recessão, principalmente para a bacia do Mocambo. Por outro lado, observa-se também que as vazões de pico entre dezembro/2009 e janeiro/2010, na bacia do riacho Tabocas, foram subestimadas. 0 Coeficiente Nash-Sutcliffe (COE) obtido para a bacia do riacho Mocambo foi de 0,6 e para a bacia do riacho Tabocas foi de 0,7. Para valores de COE acima de 0,75 o desempenho é considerado bom, para valores entre 0,36 e 0,75 é considerado aceitável e valores inferiores a 0,36 são julgados inaceitáveis (SILVA et al., 2008).

Tabela 4 - Parâmetros calibrados no modelo SWAT na simulação de vazão

\begin{tabular}{ccc}
\hline Parâmetros & Faixa de Variação & Valor Calibrado \\
\hline r_CN2 & $-0,5-0,5$ & -0.23 \\
v_ALPHA_BF & $0,0-1,0$ & 0,65 \\
V_GW_DELAY & $1,0-450$ & 200 \\
v_GWQMN & $0,0-2,0$ & 1,22 \\
v_GW_REVAP & $0,02-0,20$ & 0,2 \\
V_ESCO & $0,8-1,0$ & 0,3 \\
V_CH_N2 & $0,02-0,05$ & 0,04 \\
V_CH_K2 & $5,0-130,0$ & 57,85 \\
V_ALPHA_BNK & $0,0-130,0$ & 0,15 \\
r_SOL_AWC* & $-0,2$ a 0,4 & 0,20 \\
r_SOL_K* & $-0,8$ a 0,8 & $-0,22$ \\
r_SOL_BD* & $-5,0$ a 5,0 & 0,00 \\
\hline
\end{tabular}

Nota: r_o parâmetro é multiplicado (por 1 + valor dado); v_o parâmetro será substituído pelo valor dado.

Fonte: Elaborado pelo autor 
Figura 9 - Hidrograma observado e calibrado para a bacia hidrográfica do riacho Mocambo

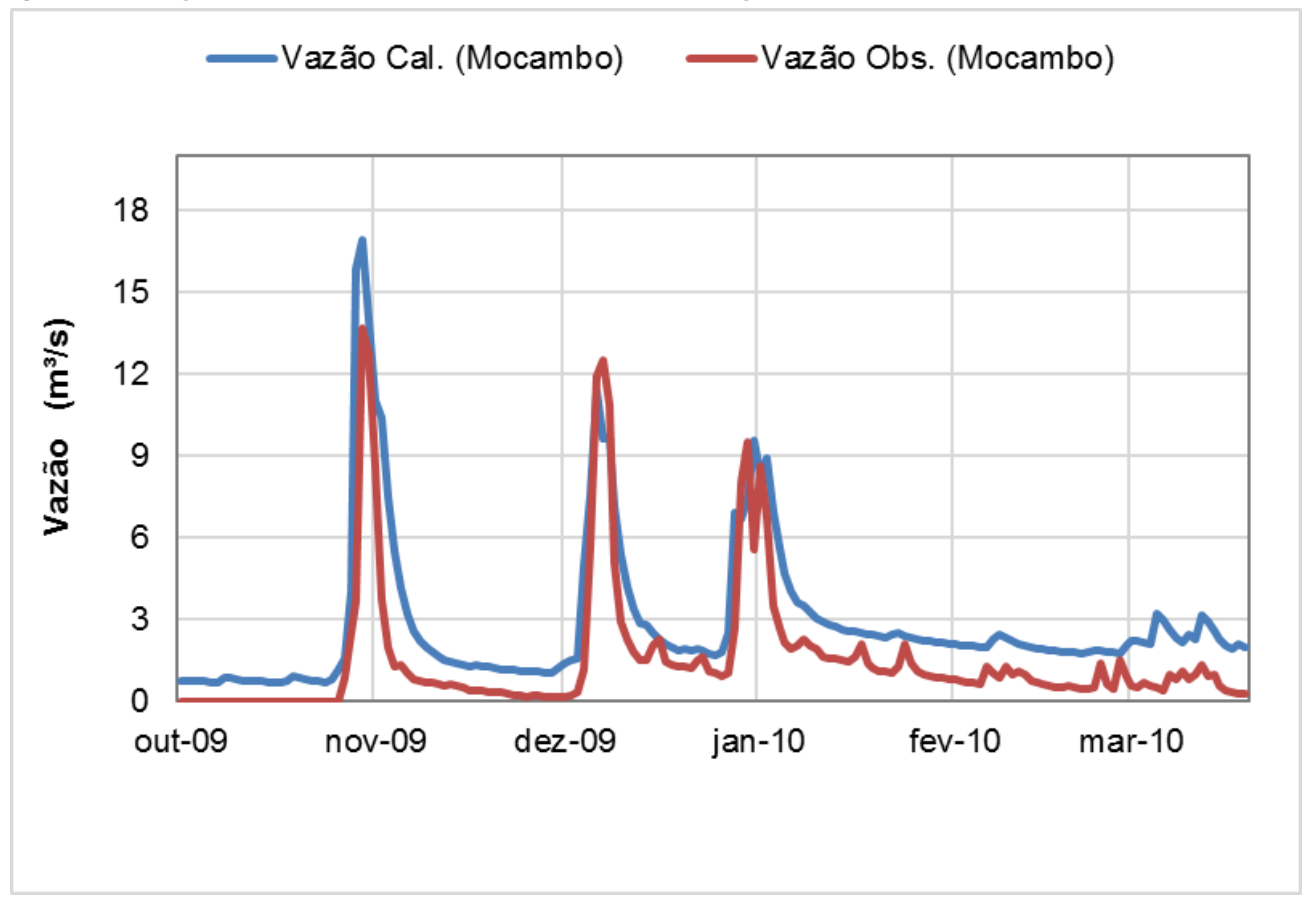

Fonte: Elaborado pelo autor

Figura 10 - Hidrograma observado e calibrado para a bacia hidrográfica do riacho Tabocas

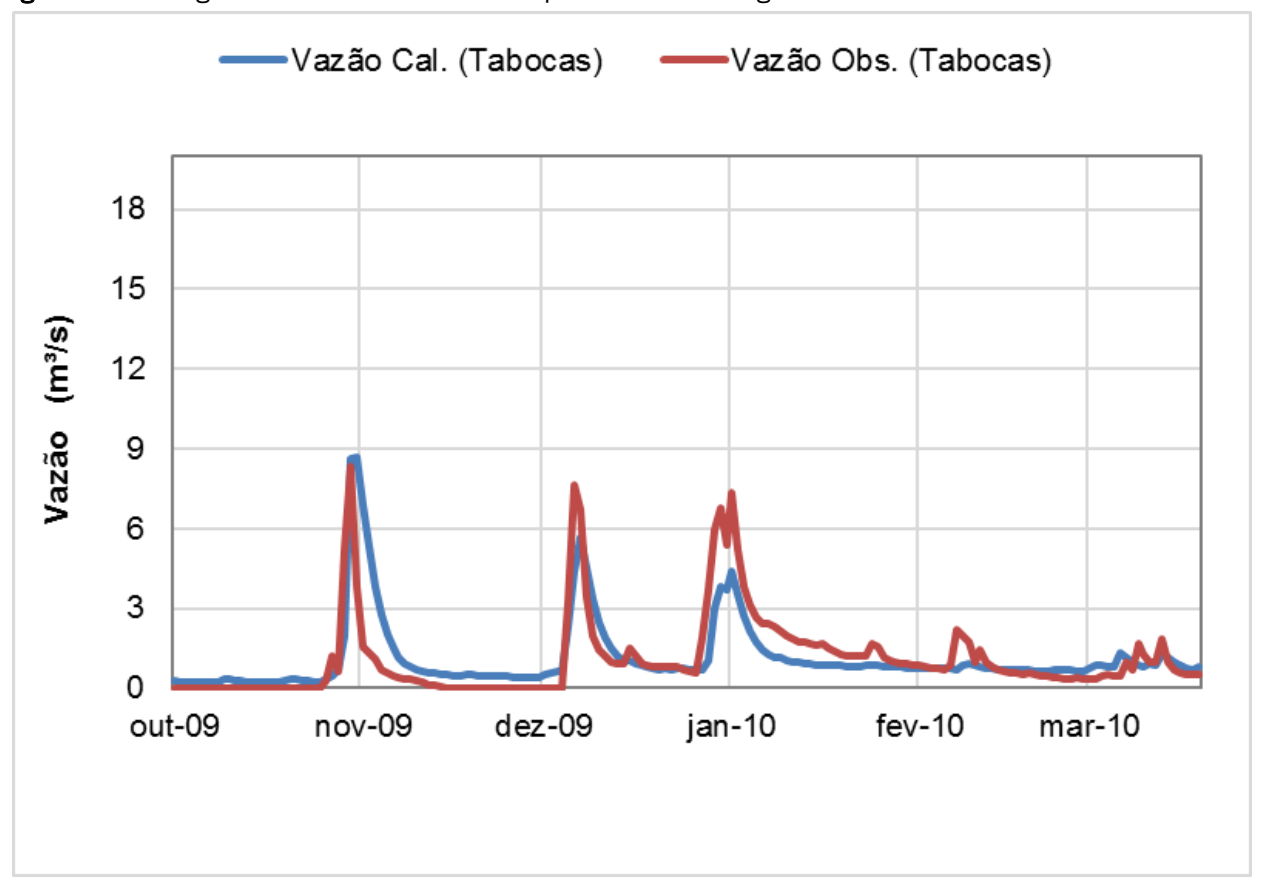

Fonte: Elaborado pelo autor

Os valores de recarga foram estimados pelo modelo SWAT por meio da infiltração no aquífero raso para cada sub-bacia (Figura 6). Os valores médios anuais de recarga para cada sub-bacia variaram de 110 a $319 \mathrm{~mm} / \mathrm{ano}$. Os dados médios anuais estão sumarizados na Tabela 5. Os valores médios obtidos por meio do SWAT são superiores aos valores identificados no modelo conceitual, os quais foram obtidos em calibrações de modelo anteriores e não levam em consideração características específicas de cada bacia, além da variação pluviométrica ao longo dos anos.

\section{CONCLUSÕES}

O banco de dados do SWAT não se mostrou adequado para seu uso em solos da região de estudo, exigindo a elaboração de um banco de dados próprio, além de um mapa de uso e ocupação do solo.

A calibração do modelo SWAT permitiu reproduzir os valores de vazões observados dos riachos Tabocas e Mocambo. Contudo, não foi possível o mesmo para o riacho Boi Morto, uma vez que os dados monitorados neste último corresponde a um período diferente ao da calibração.

A utilização de valores estimados para os parâmetros de propriedades do solo e climas, obtidos em referências bibliográficas e posteriormente calibrado, no modelo SWAT, foi satisfatória para a obtenção da recarga do aquífero. Os resultados modelados são 
consistentes aos estimados no modelo conceitual. Ademais, este modelo se mostrou útil uma vez que que se pode observar a varia- ção espacial e geográfica da recarga. Os resultados gerados podem ser utilizados na alimentação de modelos hidrogeológicos.

Tabela 5 - Resumo dos valores médios de recarga calculados pelo SWAT para as sub-bacias

\begin{tabular}{cccccccccc}
\hline Ano & $\mathbf{2 0 0 4}$ & $\mathbf{2 0 0 5}$ & $\mathbf{2 0 0 6}$ & $\mathbf{2 0 0 7}$ & $\begin{array}{c}\mathbf{2 0 0 8} \\
\mathrm{mm} / \mathbf{a n o}\end{array}$ & $\mathbf{2 0 0 9}$ & $\mathbf{2 0 1 0}$ & $\mathbf{2 0 1 1}$ & Média \\
\hline sub_1 & 230,7 & 330,0 & 306,6 & 203,3 & 169,7 & 247,4 & 197,1 & 329,0 & 251,7 \\
sub_2 & 120,5 & 187,9 & 192,4 & 159,3 & 65,7 & 132,6 & 87,8 & 210,7 & 144,6 \\
sub_3 & 163,6 & 229,2 & 211,8 & 128,5 & 137,0 & 157,5 & 130,3 & 231,4 & 173,7 \\
sub_4 & 149,4 & 205,9 & 180,1 & 111,6 & 121,7 & 129,0 & 127,2 & 215,8 & 155,1 \\
sub_5 & 98,7 & 143,3 & 141,7 & 121,6 & 49,7 & 93,2 & 62,9 & 167,3 & 109,8 \\
sub_6 & 305,5 & 397,2 & 351,7 & 168,7 & 286,2 & 273,5 & 271,5 & 387,9 & 305,3 \\
sub_7 & 256,0 & 333,6 & 289,4 & 141,2 & 237,0 & 224,3 & 216,9 & 322,3 & 252,6 \\
sub_8 & 317,6 & 412,1 & 366,8 & 170,4 & 307,0 & 287,8 & 285,8 & 402,3 & 318,7 \\
sub_9 & 169,8 & 245,0 & 232,1 & 144,1 & 141,4 & 170,4 & 140,7 & 249,1 & 186,6 \\
sub_10 & 297,5 & 387,6 & 343,0 & 160,6 & 285,2 & 265,7 & 266,2 & 378,1 & 298,0 \\
sub_11 & 226,5 & 309,9 & 292,8 & 158,5 & 209,0 & 221,2 & 202,1 & 314,5 & 241,8 \\
\hline
\end{tabular}

Fonte: Elaborado pelo autor

\section{REFERÊNCIAS}

ABBASPOUR K. C., M. VEJDANI, AND S. HAGHIGHAT. SWATCUP calibration and uncertainty programs for SWAT. In Proc. Intl. Congress on Modelling and Simulation (MODSIM'07), 1603-1609. L. Oxley and D. Kulasiri, eds. Melbourne, Australia: Modelling and Simulation Society of Australia and New Zealand. 2007.

AGÊNCIA NACIONAL DE ÁGUAS - ANA. Dados hidrológicos. Séries Históricas. Hidroweb. Disponivel em: www.hidroweb.ana.gov.br. Acesso em: 22 fev. 2016.

ALKMIM F. F., MARTINS-NETO M. A. A bacia intercratônica do São Francisco: Arcabouço estrutural e cenários evolutivos. In: PINTO C.P., MARTINS NETO M.A, Bacia do São Francisco: geologia e recursos naturais. Belo Horizonte: SBG-MG, p-30. 2001.

ARNOLD, J. G., SRINIVASAN, R., MUTTIAH, R. S., WILLIAMS, J. R. Large area hydrologic modeling and assessment part I: model development. Journal of the American Water Resources Association, Illinois, v. 34, n. 3, p. 73-89, Feb. 1998.

ARNOLD, J. G., KINIRY, J.R., SRINIVASAN, R., WILLIAMS, J. R., HANEY, E. B., NEITSCH, S.I. Soil \& Water Assessment Tool. Input/Output Documentation. Version 2012. Texas Water Resourses Institute, 2012. 650p.

BALDISSERA, G. C. Aplicabilidade do modelo de simulação hidrológica SWAT (Soil and Water Assessment Tool), para a bacia hidrográfica do Rio Cuiabá/MT, 2005. 144p.

BESSIANI, D. A., GASSMAN, P. W., FERNANDES, J. G., GARBOSSA, L., H., P. SRINIVASAN, R., BONUMÁ, N. B., MENDIONDO, E. M. Review of Soil and Water Assessment Tool (SWAT) applications in Brazil: Challenges and prospects. International Journal of Agricultural and Biological Engineering, v.o, n. 3 June, 2015.

CHOW, V. T. Open-channel hydraulics. McGraw-Hill, New York, p. 168-175, 1959.

COTA, S. D. S., Desenvolvimento de modelo conceitual e matemático do aquífero cárstico-fissural na bacia do Rio São Francisco: gestão de recursos hídricos como uma ferramenta para a minimização de fluorose dentária - Projeto Flúor. Relatório (Pós-Doutorado) - University of Waterloo. Waterloo, Canadá, 2011.

DE PAULA, R. S. Modelagem numérica de fluxo do aquífero cárstico-fissural do grupo Bambuí, bacia Boi Morto no município de São Francisco, Norte de Minas Gerais. Dissertação (Mestrado) - Instituto de Geociências, Universidade Fderal de Minas Gerais, 2012.

DOWLATABADI S., ZOMORODIAN S. M. A., Conjunctive simulation of surface water and groundwater using SWAT and MODFLOW in Firoozabad watershed. KSCE Journal of Civil Engineering, 27 March, 2015.
DUAN, Q., GUPTA, V.K., SOROOSHIAN, S. A shuffled complex evolution approach for effective and efficient optimization. J. Optimization Theory Appl., v. 76, n. 3, p. 501-521. 1993.

FREIRE, A. P. S., CASTRO, R. E. L. Mapeamento geológico na sub-bacia hidrográfica do riacho Mocambo com ênfase nas ocorrências de fluorita e sua relação com as anomalias de fluoreto na água subterrânea no Municí pio de São Francisco. Monografia (Graduação) - Universidade Federal de Minas Gerais, 2003. 94 p.

FUNDAÇÃO ESTADUAL DO MEIO AMBIENTE - FEAM. Mapa de solos do Estado de Minas Gerais: Folha 2 [mapa], 1:650.000. Belo Horizonte: FEAM, 2010.

LIERCH, S. The program pcpSTAT. User`s Manual. Berlin, 2003.

MANNING, R. On the flow of water in open channels and pipes. Transactions of the Institution of Civil Engineers of Ireland, v. 20, p. 161-207, 1891.

MCDONALD, M.G., HARBAUGH, A.W. A modular three-dimensional finitedifference ground-water flow model. Open-File Report 83-875. U.S. Geological Survey, December 28, 1983.

MOURA, F. D., SOUZA, A. M. Mapa geológico da sub-bacia do córrego Boi Morto, município de São Francisco, Minas Gerais. Monografia (Graduação) - Universidade Federal de Minas Gerais. Mapa Geológico, escala 1:60.000. 2010.

NEITSCH, S. L, ARNOLD, J. G, KINIRY J. R, WILLIAMS, J. R. Soil and water assessment tool: theoretical documentation version 2009. Temple: BRC, 2011. 541 p.

PINIEWSKI, M. Scenario-based impact assessment of global and regiona change on the semi-natural flow regime. Hamburg: Achor Academic Publishing. 2014. 206 p.

RIBEIRO, K. D., MENEZES, S. M., MESQUITA, M. G. B. F, SAMPAIO, F. M. T. Propriedades físicas do solo, influenciadas pela distribuição de poros de seis classes de solos da região de Lavras-MG. Ciência e Agrotecnologia, v. 31, p. 1167-1175, 2007.

SILVA, P.M.O.; MELLO, C.R.; SILVA, A.M., COELHO, G. Modelagem da hidrografa de cheia em uma bacia hidrográfica da região Alto Rio Grande. R. Bras. Eng. Agric. Amb., v. 12, p. 258-265, 2008.

UNIVERSIDADE FEDERAL DE VIÇOSA - UFV. Atlas digital das águas de Minas Gerais: consulta informativa: mapas de vazões específicas do Alto e Médio São Francisco em Minas Gerais. 3. ed. 2005. Disponível em: http://www.atlasdasaguas.ufv.br/alto e medio sao francisco/mapas de vazoes_especificas.html. Acesso em: 22 jan. 2017.

VELÁSQUEZ, L.M.N.; FANTINEL, L.M.; COSTA, W.D.; UHLEIN, A.; FERREIRA, E.F.E.; CASTILHO, L.S.; PAIXÃO, H.H. Origem do flúor na água subterrânea e sua relação como os casos de fluorose dental no município de São 
Francisco, Minas Gerais. Belo Horizonte: FAPEMIG. Relatório Final: Processo CRA 294/99, 2003. 138p.

VELÁSQUEZ, L. N. M., FANTINEL, L. M., FERREIRA, E. F. E., CASTILHO, L. S., UHLEIN, A., VARGAS, A. M. D., ARANHA, P. R. A. Fluorose dentária anomalias de flúor na água subterrânea no município de São Francisco,
Minas Gerais. In: SILVA, C.R. et al. (Org). Rio de Janeiro: CPRM, p. 110-117. 2006.

WIBLE, T. Enhanced watershed modeling and data analysis with a fully coupled hydrologic model and cloud-based flow analysis. Master of Science. Department of Civil and Environmental Engineering. Colorado State University. 2014 